\section{Hilft Amalgamentfernung beim oralen Lichen planus?}

\author{
Die Ätiologie von oralen lichenoiden Reaktionen im Mundbereich \\ (OLR) ist nach wie vor nicht geklärt. Diskutiert wird eine Typ-IV- \\ Allergie gegen Quecksilberverbindungen. Eine Studie untersuchte \\ den Zusammenhang zwischen OLR-Symptombesserung nach \\ Amalgamentfernung und Ergebnissen im Epikutantest.
}

$\mathrm{D}$ ie Mundläsionen von 134 Patienten wurden klinisch als OLR diagnostiziert. Außerdem erfolgten bei zahlreichen Patienten Biopsien, histologisch zeigte sich das Bild des Lichen planus (LP). Bei 119 Patienten konnten Patchtests mit Amalgam und anorganischem Quecksilber durchgeführt werden. Unabhängig von den Ergebnissen der Patchtests wurden bei 105 Patienten die Amalgamfüllungen entfernt. Als Kontrollgruppe dienten 29 Patienten, die die Amalgamentfernung verweigerten.

$8,2 \%$ der OLR-Patienten hatten auch einen Hautbefall mit LP. $27 \%$ der Patienten reagierten im Patchtest positiv auf Quecksilberverbindungen. Von der Amalgamentfernung profitierten $97,1 \%$, bei $29,5 \%$ verschwanden die Läsionen komplett. In der Kontrollgruppe ohne Amalgamentfernung besserte sich der Zustand lediglich bei $6,9 \%(p<0,05)$. Läsionen auf der Zunge sprachen viel besser an als ein Befall an anderen Stellen, bei den Patienten mit einem zusätzlichen kutanen LP war nur eine marginale Besserung festzustellen. Bei acht Patienten $(7,6 \%)$ traten durchschnittlich 14 Monate nach der Amalgamentfernung insgesamt 13 neue Läsionen auf. Patienten mit einem positiven Befund in der allergologischen Hauttestung zeigten häufiger eine komplette Abheilung, die zudem rascher erfolgte als bei den übrigen Patienten.

\title{
Sonnenschutz ist nicht gleich Hautkrebsschutz
}

\section{Sonnenschutzmittel sollten nicht nur vor Sonnenbrand, sondern auch vor Hautkrebs schützen und deshalb auch die UV-induzierte Suppression der zellvermittelten kutanen Immunreaktion als wichtigen Schritt der Hautkrebsentwicklung verringern.}

$\mathrm{B}$ ritische Forscher dokumentierten die Daten von 119 gesunden Probanden, überwiegend vom Hauttyp I oder II. Aufgeteilt in verschiedene Behandlungsgruppen wurde bei allen Probanden die Gesäßhaut mit steigenden UV-Dosen bestrahlt. Ermittelt wurden die Abhängigkeiten zwischen Bestrahlungen im UV-B- (280-320 nm) sowie im UV-A-Bereich (320-400 nm) und dem Auftreten eines Erythems beziehungsweise einer kutanen Immunreaktion mit und ohne Anwendung eines handelsüblichen Sonnenschutzmittels
(Lichtschutzfaktor 15). Als Test für die Wirkung auf die kutane Immunreaktion wurde die kontaktallergische Reaktion auf das Hapten 2,4-Dinitrochlorbenzol 24 Stunden nach der Bestrahlung herangezogen.

Das Sonnenschutzmittel absorbierte UV-Strahlung überwiegend im UVB-Bereich. Im Vergleich zu unbehandelten Kontrollen schützte das Externum sowohl vor einem Erythem als auch vor einer Immunsuppression. Allerdings war der Immunschutz weniger als halb so stark wie der Sonnenbrandschutz. Dies

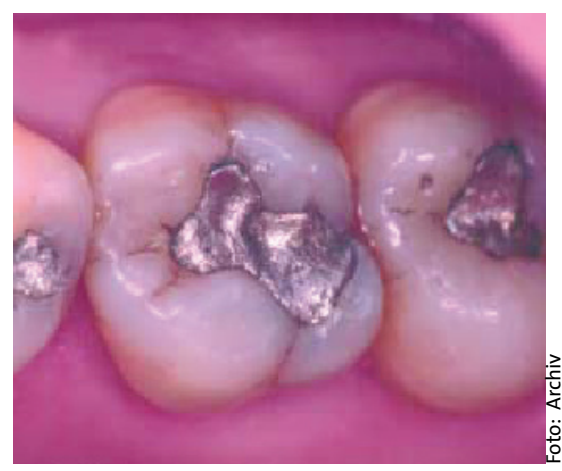

Amalgamfüllungen - wann müssen sie raus?

Fazit: Die Entfernung von Amalgamfüllungen bringt für die meisten Patienten mit einer oralen lichenoiden Reaktion eine deutliche Besserung - weitgehend unabhängig vom Befund im Patchtest. Bei Patienten mit einem zusätzlichen kutanen Lichen planus ist der Eingriff aber wenig Erfolg versprechend.

$b k$

Dunsche A et al. Oral lichenoid reactions associated with amalgam: improvement after amalgam removal. Br J Dermatol 2003; 148: 70-6

führen die Autoren auf die mangelnde Absorptionsfähigkeit des getesteten Sonnenschutzmittels im UV-A-Bereich zurück. Dieser Wellenlängenbereich ist kaum an der Sonnenbrandentstehung beteiligt, trägt aber offensichtlich maßgeblich zur UV-vermittelten kutanen Immunsuppression bei.

Fazit: Handelsübliche Sonnenschutzmittel schützen außer vor Sonnenbrand auch vor einer UV-induzierten Immunsuppression - allerdings nicht in gleichem Umfang. Einen besseren Immunschutz dürfte man bei Anwendung von Präparaten mit einem breiten UVAbsorptionsspektrum erhalten. $\quad b k$

Kelly DA et al. A commercial sunscreen's protection against ultraviolet radiationinduced immunosuppression is more than $50 \%$ lower than protection against sunburn in humans. J Invest Dermatol 2003; 120: 65-71 